\title{
Estrutura genética populacional de Copaifera langsdorffii Desf. (Leguminosae - Caesalpinioideae) em fragmentos florestais no Pontal do Paranapanema, SP, Brasil.
}

\author{
KARINA MARTINS ${ }^{1,4}$, JOÃO DAGOBERTO DOS SANTOS ${ }^{2}$, FERNANDA AMATO GAIOTTO ${ }^{3}$ \\ MARIA ANDRÉIA MORENO ${ }^{2}$ e PAULO YOSHIO KAGEYAMA ${ }^{2}$
}

(recebido: 23 de novembro de 2006; aceito: 10 de janeiro de 2008)

\begin{abstract}
Population genetic structure of Copaifera langsdorffii Desf. (Leguminosae - Caesalpinioideae) in forest fragments in "Pontal do Paranapanema", SP, Brazil). The "Pontal do Paranapanema" is the most recently deforested region of the state of São Paulo in Brazil. The effects of forest fragmentation on the population genetic structure of Copaifera langsdorffii Desf. were evaluated by genotyping with six microsatellite loci at least 30 adult and 30 sapling individuals of three populations in "Pontal do Paranapanema". The sampling areas comprised two fragments located in the rural settlements Tucano (800 ha) and Madre Cristina (300 ha); and one population located in a continuous forest, the "Parque Estadual do Morro do Diabo" (35,000 ha). High levels of genetic diversity were detected in saplings and adults, gene diversity $\left(\hat{H}_{e}\right)$ ranged from 0.718 to 0.835 and average number of alleles per locus $(\hat{A})$ ranged from 8.67 to 11.83 , showing that the fragmentation has not caused loss of alleles yet. The high estimated fixation indexes (from 0.183 to 0.387 ) are due to null alleles in some loci and the occurrence of inbreeding within populations. Genetic divergence was low among adults $\left(\hat{R}_{S T}=0.035\right)$ but increased among saplings $\left(\hat{R}_{S T}=0.075\right)$. As expected, forest fragmentation has caused a decrease in gene flow among the populations, especially between the most distant fragment (Tucano - about $8 \mathrm{~km}$ from the "Parque Estadual do Morro do Diabo"). An alternative to promote gene flow among forest fragments of "Pontal do Paranapanema" would be the settle of agroforests, which would act as stepping-stones, favouring pollen and seed dispersion among fragments.
\end{abstract}

Key words - Atlantic forest, forest fragmentation, gene flow, genetic diversity, microsatellites

RESUMO - (Estrutura genética populacional de Copaifera langsdorffii Desf. (Leguminosae - Caesalpinioideae) em fragmentos florestais no Pontal do Paranapanema, SP, Brasil) O Pontal do Paranapanema é a região mais recentemente desflorestada do Estado de São Paulo. Os efeitos da fragmentação florestal na estrutura genética de Copaifera langsdorffii Desf. foram avaliados por meio da genotipagem, com seis locos microssatélites, de pelo menos 30 indivíduos regenerantes e 30 adultos em três áreas do Pontal do Paranapanema. A amostragem compreendeu dois fragmentos localizados em assentamentos rurais, Tucano (800 ha) e Madre Cristina (300 ha); e uma população localizada em floresta contínua, no Parque Estadual do Morro do Diabo (35.000 ha). Níveis elevados de diversidade genética foram detectados tanto nos regenerantes como nos adultos. A diversidade gênica $\left(\hat{H}_{e}\right)$ variou de 0,718 a 0,835 e o número médio de alelos por loco $(\hat{A})$ variou de 8,67 a 11,83, mostrando que a fragmentação ainda não causou perda de alelos. As estimativas elevadas dos índices de fixação $(0,183$ a 0,387$)$ foram consequientes da ocorrência de alelos nulos em alguns locos e de endogamia nas populações. A divergência genética foi pequena entre os adultos $\left(\hat{R}_{S T}=0,035\right)$, mas aumentou entre os regenerantes $\left(\hat{R}_{S T}=0,075\right)$. Como esperado, a fragmentação florestal está causando redução do fluxo gênico interpopulacional, especialmente em relação ao fragmento mais distante da floresta contínua (Tucano - cerca de $8 \mathrm{~km}$ ). Uma alternativa para promover o fluxo gênico entre os remanescentes florestais do Pontal do Paranapanema seria o estabelecimento de sistemas agroflorestais pelos agricultores assentados, os quais atuariam como trampolins ecológicos, favorecendo a dispersão de pólen e sementes entre fragmentos.

Palavras-chave - diversidade genética, Floresta Atlântica, fluxo gênico, fragmentação florestal, microssatélites

\section{Introdução}

A fragmentação florestal reduz áreas de vegetação contínua em paisagens compostas por uma matriz de

\footnotetext{
1. Empresa Brasileira de Pesquisa Agropecuária, Centro de Pesquisa Agroflorestal do Acre, Rodovia BR 364, km14, Caixa Postal 321, 69908-970 Rio Branco, AC, Brasil.

2. Escola Superior de Agricultura Luiz de Queiroz, Departamento de Ciências Florestais, Av. Pádua Dias, 11, Caixa Postal 09, 13418-900 Piracicaba, SP, Brasil.

3. Universidade Estadual de Santa Cruz, Departamento de Ciências Biológicas, Genética e Biologia Molecular, Rodovia Ilhéus-Itabuna, km 16, Salobrinho, 45662-000 Ilhéus, BA, Brasil.

4. Autor para correspondência: karimartins@yahoo.com
}

pastagens e agricultura entremeada com fragmentos florestais de diversos formatos e tamanhos. Do ponto de vista da conservação genética, a supressão de extensas áreas florestadas causa uma diminuição nos tamanhos populacionais, podendo resultar na perda imediata de alelos (Young et al. 1996). Se populações fragmentadas permanecem pequenas e isoladas por muitas gerações, poderá haver um aumento da endogamia, da deriva genética e da divergência genética entre populações, devido à ocorrência de cruzamentos entre os poucos indivíduos remanescentes e à limitação do fluxo de genes via pólen e sementes entre os fragmentos. A principal 
consequiência desses processos é a redução da diversidade genética. Em último caso, a perda de diversidade genética pode resultar na redução na adaptabilidade de populações remanescentes e na extinção da espécie (England et al. 2002).

A sobrevivência e manutenção de populações fragmentadas de espécies arbóreas dependerão da definição de estratégias adequadas de manejo dos remanescentes florestais. Para que essas estratégias sejam efetivas, é fundamental primeiramente entender e quantificar os principais efeitos populacionais e genéticos da fragmentação. Para tal, a estratégia de comparar a estrutura genética entre populações de adultos nos fragmentos com populações situadas em áreas de vegetação contínua preservada (população controle) permite identificar se os padrões genéticos observados nas populações fragmentadas são resultantes da diminuição da população original, ou são indicativos de níveis naturais esperados para a espécie (Williams et al. 2003). Essa estratégia possibilita também inferir sobre os efeitos imediatos da fragmentação florestal, resultantes da perda de indivíduos pela redução de habitat. Esse tipo de abordagem tem sido adotado em alguns trabalhos (e.g. Young et al. 1993, Aldrich et al. 1998, Dayanandan et al. 1999). Adicionalmente, a avaliação da diversidade genética de indivíduos juvenis, que estão regenerando nos fragmentos, e sua comparação com a diversidade observada em indivíduos adultos indicarão os efeitos genéticos da fragmentação em curto e em médio prazo, resultantes da alteração nos padrões de fluxo gênico e dos efeitos de deriva genética, endogamia e seleção.

Copaifera langsdorffii Desf. (Leguminosae, Caesalpinioideae) é uma espécie arbórea tropical amplamente distribuída no Brasil $\left(03^{\circ}\right.$ a $24^{\circ} 50^{\prime} \mathrm{S}$ de latitude), sendo encontrada nas florestas Amazônica e Atlântica e nas matas ciliares da região do cerrado. No Estado de São Paulo, a maioria das populações encontra-se fragmentada.

As flores são pequenas (cerca de $0,5 \mathrm{~cm}$ de diâmetro), apétalas e reunidas em inflorescências (Freitas \& Oliveira 2002). Produzem néctar e grande quantidade de pólen e são polinizadas por abelhas como Trigona sp. e Apis mellifera (Crestana \& Kageyama 1989, Freitas \& Oliveira 2002). Os indivíduos florescem durante a estação chuvosa, sendo que no Estado de São Paulo a floração é mais intensa nos meses de dezembro a fevereiro (Predroni et al. 2002). O florescimento é regular, mas supra-anual, sendo que o número de árvores que florescem em cada estação reprodutiva é variável (Crestana \& Kageyama 1989, Pedroni et al. 2002). A espécie é hermafrodita e os frutos são predominatemente formados após polinização cruzada (Freitas \& Oliveira 2002).
A frutificação ocorre durante a estação seca (de abril a setembro). A taxa de conversão flor-fruto é baixa, devido à polinização ineficiente e à predação de frutos (Freitas \& Oliveira 2002). A dispersão das sementes é realizada por aves como o tucano (Ramphastos toco, Rhamphastidae) e a gralha-do-campo (Cyanocorax cristatellus, Corvidae), que engolem o arilo e regurgitam as sementes (Motta Junior \& Lombardi 1990) e também por hidrocoria, devido à ocorrência freqüente ao longo de cursos de água. C. langsdorffii é produtora de óleo-resina, o qual é encontrado em canais secretores localizados em todas as partes da árvore, especialmente no tronco. As utilizações do óleo de copaíba na medicina popular são muitas e indicam uma grande variedade de propriedades farmacológicas, sendo que as principais atividades relatadas foram de antiinflamatório das vias superiores e inferiores e cicatrizante (Veiga Júnior \& Pinto 2002). O óleo-resina pode ser utilizado puro (in natura ou destilado), ou como componente na preparação de produtos terapêuticos e cosméticos; possui ainda potencial para uso industrial em tintas, vernizes e como fixador de fragrância de perfumes (Sampaio 2000).

O objetivo desse estudo foi avaliar a estrutura genética populacional de indivíduos regenerantes e adultos de Copaifera langsdorffii Desf. em fragmentos florestais da região do Pontal do Paranapanema, extremo-oeste do Estado de São Paulo.

\section{Material e métodos}

Área de estudo e amostragem - Foram selecionadas três áreas para coleta de material vegetal de C. langsdorffii na região do Pontal do Paranapanema, extremo-oeste do Estado de São Paulo. Devido ao processo predatório de ocupação, atualmente restam apenas cerca de $1,85 \%$ da vegetação original, constituída por floresta sazonal semidecídua. A maior área contínua remanescente florestal está no Parque Estadual do Morro do Diabo (35.000 ha) e há vegetação remanescente em alguns fragmentos em áreas particulares (Dean 1996), que totalizam aproximadamente 15.000 ha. Duas populações foram amostradas em fragmentos situados em assentamentos de Reforma Agrária e uma população foi coletada em área de floresta contínua e preservada, no Parque Estadual do Morro do Diabo (MD). O fragmento Tucano (TU) (800 ha) dista cerca de $8 \mathrm{~km}$ do MD e o fragmento Madre Cristina (MC) (320 ha) é separado do MD pela largura de uma estrada. Em cada área de coleta (tabela 1), foram amostrados e georreferenciados 30 indivíduos adultos contíguos (DAP > $30 \mathrm{~cm}$ ). Dentro do perímetro determinado pelas 30 árvores adultas foram instaladas cinco parcelas aleatórias de $100 \mathrm{~m}^{2}$, para amostragem dos regenerantes. Como os parâmetros de diversidade genética são sensíveis ao efeito da amostragem (Leberg 2002), nessas parcelas, amostrou-se 
pelo menos 30 indivíduos regenerantes com altura entre 0,5 e 2,0 m para que fosse possível a comparação da diversidade genética com a classe de adultos. O número total de regenerantes amostrados em cada população foi variável (tabela 1). As amostras foliares dos indivíduos amostrados foram secas em sílica gel e transportadas ao Laboratório de Reprodução e Genética de Espécies Arbóreas (LARGEA), localizado no Departamento de Ciências Florestais da Escola Superior de Agricultura Luís de Queiroz (ESALQ-USP), em Piracicaba - SP, onde as análises laboratoriais foram conduzidas.

Tabela 1: Número de adultos (árvores com DAP $>30 \mathrm{~cm}$ ) e regenerantes $(0,5$ a 2,0 m de altura) amostrados nas três áreas de coleta e localização geográfica das populações de Copaifera langsdorffii. (MD = Parque Estadual do Morro do Diabo; $\mathrm{MC}=$ fragmento Madre Cristina; $\mathrm{TU}=$ fragmento Tucano).

Table 1: Number of adults (tree with $\mathrm{DBH}>30 \mathrm{~cm})$ and saplings $(0.5-2.0 \mathrm{~m}$ height) sampled in the three sample sites and spatial location of Copaifera langsdorffii populations. (MD = "Parque Estadual do Morro do Diabo"; MC = "Madre Cristina"; TU = "Tucano").

\begin{tabular}{|c|c|c|c|c|}
\hline \multirow[t]{2}{*}{ População } & \multicolumn{2}{|c|}{ Localização geográfica } & \multirow[t]{2}{*}{ Adultos } & \multirow[t]{2}{*}{ Regenerantes } \\
\hline & Latitude & Longitude & & \\
\hline MD & $22^{\circ} 25^{\prime} 32^{\prime \prime} \mathrm{S}$ & $52^{\circ} 22^{\prime} 17^{\prime \prime} \mathrm{W}$ & 30 & 28 \\
\hline $\mathrm{MC}$ & $22^{\circ} 28^{\prime} 32^{\prime \prime} \mathrm{S}$ & $52^{\circ} 28^{\prime} 37^{\prime \prime} \mathrm{W}$ & 30 & 34 \\
\hline $\mathrm{TU}$ & $22^{\circ} 28^{\prime} 38^{\prime \prime} \mathrm{S}$ & $52^{\circ} 28^{\prime} 33^{\prime \prime} \mathrm{W}$ & 30 & 29 \\
\hline
\end{tabular}

Procedimento Laboratorial - A extração do DNA genômico total das amostras foliares foi realizada seguindo o protocolo CTAB descrito por Doyle \& Doyle (1987). Os indivíduos foram genotipados com seis locos microssatélites (Ciampi et al. 2000). O coquetel $(13 \mu \mathrm{L})$ para a realização da PCR foi composto por 15,0 ng de DNA genômico, $250 \mu \mathrm{M}$ de dNTPs, 0,5 $\mu \mathrm{M}$ de $\mathrm{MgCl}_{2}$, tampão para PCR 1X (10 mM de Tris- $\mathrm{HCl}, 50 \mathrm{mM}$ de $\mathrm{KCl}, 1,5 \mathrm{mM}$ de $\mathrm{MgCl}_{2}, \mathrm{pH} 8,3$ ), $2,5 \mu \mathrm{g} \mathrm{mL}^{-1}$ de BSA, 0,2 $\mu \mathrm{M}$ de cada iniciador e $1 \mathrm{U}$ de Taq DNA polimerase (Phoneutria). As amplificações foram realizadas em termociclador do tipo MJ Research PTC-100, utilizando o seguinte protocolo: $96{ }^{\circ} \mathrm{C}$ por 2 minutos; 30 ciclos de $94{ }^{\circ} \mathrm{C}$ por 1 minuto, temperatura de hibridação específica de cada par de iniciadores por 1 minuto, $72{ }^{\circ} \mathrm{C}$ por 1 minuto e terminando com $72{ }^{\circ} \mathrm{C}$ por 7 minutos. Após a amplificação, os fragmentos de DNA foram separados em gel desnaturante de poliacrilamida a $4 \%$, em corrida de uma hora em tampão TBE $1 \mathrm{X}$ em cuba vertical. Os fragmentos foram observados na forma de bandas, após coloração com nitrato de prata, seguindo o protocolo Creste et al. (2001). O tamanho dos alelos foi determinado por comparação com um marcador de peso molecular padrão (10-pb "ladder" - Invitrogen $\left.{ }^{\circledR}\right)$. Fragmentos amplificados de diferentes tamanhos foram considerados alelos diferentes.

Análise estatística - As frequiências alélicas em cada loco foram utilizadas para estimar diversidade gênica $\left(\hat{H}_{e}\right)$, com uso do programa GDA (Lewis \& Zaykin 2000). Com o número observado de alelos $(\hat{A})$ estimou-se a máxima diversidade possível com a equação $\hat{h}_{\max }=(\hat{A}-1) / \hat{A}$. A magnitude da diversidade gênica foi quantificada por meio da comparação entre a diversidade gênica estimada $\left(\hat{H}_{e}\right)$ e a máxima diversidade possível em cada loco $\left(\hat{h}_{\max }\right)$. Desvios das proporções esperadas pelo Equilíbrio de Hardy-Weinberg
(EHW) em cada loco foram avaliadas por meio do teste $\chi^{2}$ com uso do programa CERVUS 2.0 (Marshall et al. 1998). A identificação de erros de genotipagem, devido à existência de alelos nulos e a erros na interpretação dos géis, foi verificada com uso do programa Micro-Checker Versão 2.2.3 (Oosterhout et al. 2004). A frequiência de alelos nulos em cada loco foi estimada como em Brookfield (1996), usando o estimador no $1: \hat{r}=\left(\hat{H}_{e}-\hat{H}_{0}\right) /\left(1+\hat{H}_{e}\right)$

Estimativas médias de diversidade gênica $\left(\hat{H}_{e}\right)$, número médio de alelos por loco $(\hat{A})$ e índice de fixação $\left(\hat{f=1}-\hat{H}_{0} / \hat{H}_{e}\right)$, bem como o número de alelos exclusivos $\left(\hat{A}_{p}\right)$, foram obtidos para as classes de regenerantes e adultos de cada população. O intervalo de confiança a $99 \%$ do $\hat{f}$ foi obtido por 10.000 reamostragens "bootstrap" sobre locos, com o programa GDA (Lewis \& Zaykin 2000). O programa TFPGA 1.3 (Miller 1997) foi utilizado para calcular as distâncias genéticas de Nei (1978) entre populações e entre regenerantes e adultos de uma mesma população e para construir um dendrograma utilizando o método de agrupamento UPGMA. A consistência dos nós do dendrograma foi avaliada por 10.000 reamostragens "bootstrap" sobre locos, com uso do software BOOD v. 2.0 (Coelho 2000).

A divergência genética entre pares de populações para ambas as classes de tamanho foi acessada usando o parâmetro $\hat{R}_{S T}$ (Goodman 1997). O número de migrantes por geração $(\mathrm{Nm})$ entre populações foi calculado pelo método indireto, com uso da fórmula $N m=1 / 4\left(1 / \hat{F}_{S T}-1\right)$ (Wright 1951). Nos cálculos, parâmetro $\hat{F}_{S T}$ foi substituído por $\hat{R}_{S T}$. Intervalos de confiança a $95 \%$ de $\hat{R}_{S T}$ foram obtidos por 1.000 reamostragens "bootstrap" sobre locos. Testes de permutação foram realizados para determinar se os valores observados de $\hat{R}_{S T}$ diferiam significativamente de zero. As análises foram realizadas com o programa $\mathrm{R}_{\mathrm{ST}}$ Calc 2.2 (Goodman 1997). 


\section{Resultados e discussão}

Diversidade genética dos locos - Nos 181 indivíduos genotipados (90 adultos e 91 regenerantes) com seis locos microssatélites foram identificados 108 alelos. Todos os seis locos foram altamente polimórficos, com 13 alelos no loco menos variável e 22 no mais variável. A diversidade gênica $\left(\hat{H}_{e}\right)$ alcançou níveis superiores a $80 \%$ em todos os locos (tabela 2). Os locos CL20 e CL34 foram os que apresentaram a diversidade genética mais elevada, uma vez que a diversidade gênica $\left(\hat{H}_{e}\right)$ representou mais de $95 \%$ da diversidade total possível de acordo com o número de alelos (tabela 2).

Em todos os locos, o número observado de heterozigotos foi menor que o esperado pelas proporções do EHW, sendo que apenas para os locos CL06 e CL20 os desvios não foram significativos. Em locos microssatélites, a ocorrência de alelos nulos pode causar desvios no EHW, levando a conclusões erradas sobe a diversidade genética das populações. Alelos nulos são alelos que consistentemente não são amplificados na PCR, e, portanto, não são detectados quando os indivíduos são genotipados. A consequiência é a detecção errônea de quantidade maior de genótipos homozigóticos em populações avaliadas por locos microssatélites (Aldrich et al. 1998, White et al. 1999). Alelos nulos têm sido encontrados em mais de $25 \%$ dos locos de microssatélites, chegando a atingir freqüências superiores a 15\% (Jarne \& Lagoda 1996).

A frequiência de alelos nulos nos locos CL02 e CL34 foi significativa para regenerantes e adultos de todas as populações estudadas (tabela 3). O loco CL27 também apresentou freqüência significativa de alelos nulos para a maioria das populações em ambas as classes de regenerantes e adultos. Portanto, o déficit no número de heterozigotos em cada loco foi decorrente, em grande parte, da ocorrência de alelos nulos (tabela 3 ).

Diversidade dentro de classes de tamanho e de populações - As estimativas de diversidade gênica $\left(\hat{H}_{e}\right)$ e número médio de alelos por loco $(\hat{A})$ não diferiram entre as classes de adultos e regenerantes estudadas (tabela 4). Observou-se uma tendência de menor diversidade genética nos indivíduos regenerantes do fragmento Tucano. Essa diferença, porém, não foi significativa. Os indivíduos regenerantes do fragmento Madre Cristina foram os que apresentaram o maior número de alelos exclusivos $\left(\hat{A}_{p}=6\right)$, indicando que propágulos vindos de populações não amostradas estão colonizando essa área. O mesmo pode ser observado nos indivíduos regenerantes do Parque Estadual Morro do Diabo, onde foram encontrados quatro alelos exclusivos (tabela 4).

Os regenerantes e adultos de C. langsdorffii estudados apresentaram níveis elevados de diversidade genética, como esperado para marcadores altamente polimórficos, como os microssatélites. Níveis semelhantes de diversidade genética foram observados em um estudo com populações adultas de C. langsdorffii na Região Centro-Oeste do Brasil (Ciampi \& Grattapaglia 2001).

Na região do Pontal do Paranapanema, a fragmentação florestal e o abate seletivo de árvores não resultaram em perda significativa de alelos nos adultos. De fato, embora duas populações estudadas encontrem-se em fragmentos, esses são de tamanho grande e a perda de indivíduos

Tabela 2. Parâmetros de diversidade genética estimados para cada loco microssatélite. Unidade de repetição de cada loco microssatélite, Temperatura de hibridação $\left(\mathrm{T}_{\mathrm{m}}\right)$, número de alelos $(\hat{A})$, amplitude alélica, diversidade gênica $\left(\hat{H}_{e}\right)$, máxima diversidade gênica possível $\left(\hat{h}_{\max }\right)$, proporção da máxima diversidade possível $\left(\hat{H}_{e} / \hat{h}_{\max }\right)$, desvios das proporções do Equilíbrio de Hardy-Weinberg (EHW).

Table 2. Parameters of genetic diversity estimated with each microsatellite locus. Repeat motif, annealing temperature $\left(\mathrm{T}_{\mathrm{m}}\right)$, number of alleles $(\hat{A})$, allele range, gene diversity $\left(\hat{H}_{e}\right)$, maximum possible diversity $\left(\hat{h}_{\text {max }}\right)$, proportion of the maximum diversity $\left(\hat{H}_{e} / \hat{h}_{m a x}\right)$ and deviations in Hardy-Weinberg proportions (EHW).

\begin{tabular}{lcccccccc}
\hline Loco & $\begin{array}{c}\text { Unidade de } \\
\text { repetição }\end{array}$ & $\begin{array}{c}\mathrm{T}_{\mathrm{m}} \\
\left({ }^{\circ} \mathrm{C}\right)\end{array}$ & $\hat{A}$ & $\begin{array}{c}\text { Amplitude } \\
\text { alélica }\end{array}$ & $\hat{H}_{e}$ & $\hat{h}_{\max }$ & $\begin{array}{c}\hat{H}_{e} / \hat{h}_{\text {max }} \\
(\%)\end{array}$ & $\begin{array}{c}\mathrm{EHW} \\
\text { CL 01 }\end{array}$ \\
\hline (AG) $)_{24}$ & 56 & 18 & $172-210$ & 0,842 & 0,944 & 89,19 & $* *$ \\
CL 02 & $(\mathrm{TC})_{25}$ & 56 & 18 & $252-298$ & 0,826 & 0,944 & 87,50 & $* *$ \\
CL 06 & $(\mathrm{TC})_{14}$ & 54 & 20 & $128-178$ & 0,798 & 0,950 & 84,00 & $\mathrm{~ns}$ \\
CL 20 & $(\mathrm{AG})_{21}$ & 56 & 22 & $092-136$ & 0,909 & 0,955 & 95,18 & $\mathrm{~ns}$ \\
CL 27 & $(\mathrm{TC})_{25}$ & 54 & 16 & $172-200$ & 0,837 & 0,937 & 89,33 & $* *$ \\
CL 34 & $(\mathrm{AG})_{33}$ & 50 & 13 & $182-208$ & 0,879 & 0,923 & 95,23 & $* *$ \\
\hline
\end{tabular}

ns = não significativo (not significant); $* *$ = significativo a $1 \%$ (significant at $1 \%)$. 
Tabela 3. Frequiência de alelos nulos em cada loco (estimador 1 de Brookfield 1996), para classes de regenerantes e adultos em três populações de Copaifera langsdorffii no Pontal do Paranapanema, SP. (MD = Parque Estadual do Morro do Diabo; $\mathrm{MC}=$ Madre Cristina; TU $=$ Tucano) .

Table 3: Null alleles frequency in each locus (estimator 1, Brookfield 1996), in saplings and adults of three Copaifera langsdorffii populations in "Pontal do Paranapanema", SP. (MD = "Parque Estadual do Morro do Diabo", MC = "Madre Cristina"; TU = "Tucano").

\begin{tabular}{|c|c|c|c|c|c|c|}
\hline & \multicolumn{3}{|c|}{ Adultos } & \multicolumn{3}{|c|}{ Regenerantes } \\
\hline & $\mathrm{MC}$ & MD & $\mathrm{TU}$ & $\mathrm{MC}$ & MD & $\mathrm{TU}$ \\
\hline CL01 & $0,0436^{\mathrm{ns}}$ & $0,0112^{\mathrm{ns}}$ & $0,1954^{* *}$ & $0,0855^{* *}$ & $0,2306^{* *}$ & $0,0743^{\mathrm{ns}}$ \\
\hline CL02 & $0,1796^{* *}$ & $0,1241^{*}$ & $0,2180^{* *}$ & $0,1386^{* *}$ & $0,1626^{* * *}$ & $0,2118^{* *}$ \\
\hline CL06 & $0,1597^{* *}$ & $0,0163^{\mathrm{ns}}$ & $0,0677^{\mathrm{ns}}$ & $-0,0267^{\mathrm{ns}}$ & $-0,0029^{\mathrm{ns}}$ & $-0,0838^{\mathrm{ns}}$ \\
\hline CL20 & $0,1478^{* *}$ & $0,1087^{*}$ & $0,0940^{\mathrm{ns}}$ & $-0,0572^{\mathrm{ns}}$ & $0,0029^{\mathrm{ns}}$ & $0,0468^{\mathrm{ns}}$ \\
\hline CL27 & $0,3754^{* *}$ & $0,3282^{* *}$ & $0,2625^{* *}$ & $0,2289^{* *}$ & $0,2862^{* *}$ & $-0,0485^{\mathrm{ns}}$ \\
\hline CL34 & $0,1564^{* *}$ & $0,1244^{* *}$ & $0,1184^{*}$ & $0,1404^{* *}$ & $0,1230^{*}$ & $0,2734^{* *}$ \\
\hline
\end{tabular}

ns = não significativo (not significant) $*$ = significativo a $5 \%($ significant at $5 \%) ; *$ * = significativo a $1 \%($ significant at $1 \%)$.

adultos não foi tão drástica a ponto de causar perda imediata e significativa de diversidade genética. Uma vez que são justamente os adultos remanescentes os que geram os propágulos que formarão as próximas gerações, os alelos mantidos nas populações adultas contribuirão para a manutenção da diversidade alélica na regeneração da espécie.

O índice de fixação dos indivíduos regenerantes dos fragmentos Madre Cristina $(\hat{f}=0,197)$ e Tucano $(\hat{f}=0,183)$ não diferiram estatisticamente de zero. Nas demais populações, observou-se um significativo déficit de indivíduos heterozigóticos em relação ao esperado pelo EHW, resultando em estimativas elevadas de $\hat{f}$. Como apresentado anteriormente (tabela 3), acredita-se que esses valores sejam decorrentes não apenas da ocorrência de endogamia nas populações como também da presença de alelos nulos em alguns locos. Em um estudo com populações de espécies arbóreas, Aldrich et al. (1998) constataram que o déficit de genótipos heterozigóticos era tipicamente maior nos locos de microssatélites com maior polimorfismo. A maior taxa de mutação nesses locos pode resultar tanto em maior

Tabela 4. Parâmetros de diversidade genética estimados com seis locos microssatélites, em regenerantes e adultos de três populações de Copaifera langsdorffii no Pontal do Paranapanema, SP. Tamanho amostral $(n)$, diversidade gênica $\left(\hat{H}_{e}\right)$, número médio de alelos por loco $(\hat{A})$, número de alelos exclusivos $\left(\hat{A}_{p}\right)$ e índice de fixação $(\hat{f})$. Erros-padrão entre parenteses. $(\mathrm{MD}=$ Parque Estadual do Morro do Diabo; MC = Madre Cristina; TU = Tucano).

Table 4. Genetic diversity parameters estimated with six microsatellites loci, in saplings and adults from three Copaifera langsdorffii populations in "Pontal do Paranapanema", SP. Sample size $(n)$, gene diversity $\left(\hat{H}_{e}\right)$, average number of alleles per locus $(\hat{A})$, number of private alleles $\left(\hat{A}_{p}\right)$ and fixation index $(\hat{f})$. Standard errors are in parentheses. $(\mathrm{MD}=$ "Parque Estadual do Morro do Diabo", MC = "Madre Cristina"; TU = "Tucano").

\begin{tabular}{llccccc}
\hline População & Classes de tamanho & $n$ & $\hat{H}_{e}$ & $\hat{A}$ & $\hat{A}_{p}$ & $\hat{f}\left(\mathrm{IC}_{99 \%}\right)^{1}$ \\
\hline \multirow{2}{*}{ MD } & Adultos & $28,33(0,23)$ & $0,806(0,014)$ & $10,33(1,05)$ & 2 & $0,278(0,086 ; 0,564)$ \\
& Regenerantes & $23,00(0,80)$ & $0,780(0,040)$ & $9,83(1,25)$ & 4 & $0,351(0,119 ; 0,612)$ \\
\multirow{2}{*}{ MC } & Adultos & $25,17(1,73)$ & $0,760(0,035)$ & $10,00(1,26)$ & 1 & $0,387(0,175 ; 0,642)$ \\
& Regenerantes & $32,00(0,49)$ & $0,794(0,038)$ & $11,83(1,22)$ & 6 & $0,197(-0,037 ; 0,441)$ \\
TU & Adultos & $28,33(0,92)$ & $0,835(0,018)$ & $10,83(0,96)$ & 4 & $0,361(0,214 ; 0,506)$ \\
& Regenerantes & $23,33(1,91)$ & $0,718(0,054)$ & $8,67(1,83)$ & 1 & $0,183(-0,104 ; 0,525)$ \\
\hline
\end{tabular}

Intervalo de confiança (IC) a 99\%, obtido por 10.000 reamostragens "bootstrap" sobre locos (Confidence interval (IC) at $99 \%$, trough 10,000 bootstraps over loci). 
número de alelos, como na maior freqüência de alelos nulos. As estimativas elevadas de índices de fixação podem ser conseqüentes também de cruzamentos não aleatórios e ocorrência de endogamia dentro dos fragmentos. As estimativas obtidas nesse estudo são comparáveis ao observado em outros locos microssatélites desenvolvidos para espécies arbóreas tropicais (Chase et al. 1996, Aldrich et al. 1998) bem como estudos com espécies arbóreas realizados com isoenzimas (Shapcott 1998). Diversidade entre classes de tamanho e populações Os cálculos das distâncias de Nei (1978) mostram que as distâncias genéticas entre regenerantes e adultos de um mesmo local são maiores que as distâncias genéticas entre as populações dos três locais. Enquanto as distâncias de Nei entre os adultos das três áreas estão próximas de 0,15 (figura 1), a distância entre regenerantes e adultos de um mesmo local atinge valores superiores a 0,45 . Os regenerantes do fragmento Tucano divergem tanto dos adultos quanto dos regenerantes dos outros locais (figura 1).

A maior diferenciação genética dos regenerantes do fragmento Tucano é confirmada pelas estimativas de $\hat{R}_{S T}$ entre pares de populações (tabela 5). Enquanto nos adultos a diferenciação genética entre as populações do Parque Estadual do Morro do Diabo (MD) e do fragmento Tucano $\left(\hat{R}_{S T}=0,0157\right)$ não tenha sido estatisticamente significativa, entre os regenerantes desses mesmos dois locais foi observada elevada diferenciação genética $\left(\hat{R}_{S T}=0,091\right)$. Observou-se também um aumento da diferenciação genética entre os regenerantes de Tucano e de Madre

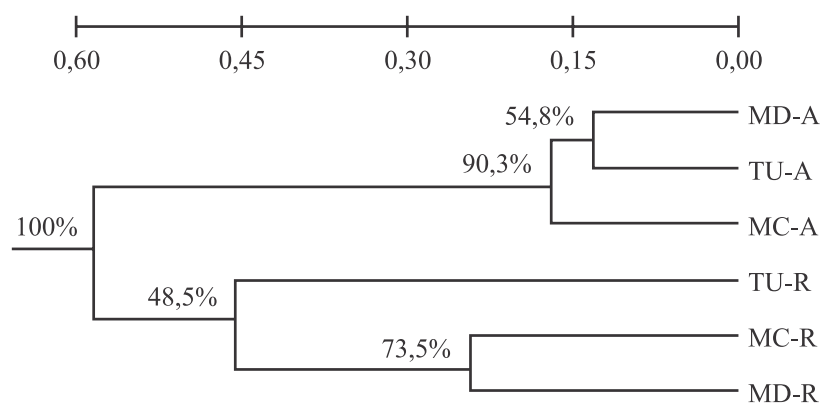

Figura 1. Dendrograma obtido por agrupamento UPGMA, a partir das distâncias genéticas de Nei (1978) entre pares de classes de regenerantes (-R) e adultos (-A) de três populações de Copaifera langsdorffii no Pontal do Paranapanema, SP. $(\mathrm{MD}=$ Parque Estadual Morro do Diabo, $\mathrm{MC}=$ Madre Cristina e TU $=$ Tucano).

Figure 1. Dendrogram defined by the UPGMA clustering, from Nei (1978) genetic distances between pairs of saplings (-R) and adults (-A) in three Copaifera langsdorffii populations in "Pontal do Paranapanema", SP. (MD = "Parque Estadual do Morro do Diabo"; MC = "Madre Cristina"; TU = "Tucano").
Cristina $\left(\hat{R}_{S T}=0,100\right)$, em relação à diferenciação entre os adultos $\left(\hat{R}_{S T}=0,037\right)$ dessas duas populações. Considerando o conjunto de populações, o $\hat{R}_{S T}$ entre os adultos foi estimado em 0,035 (I.C.: 0,017 a 0,096) e entre os regenerantes 0,076 (I.C.: 0,056 a 0,134).

Estudos de simulação (Carlini-Garcia et al. 2001, Kalinowski 2002, Cervantes-Martinez et al. 2006) e revisão (Balloux \& Lugon-Moulin 2002) têm mostrado que locos altamente polimórficos, como os marcadores microssatélites utilizados no presente estudo, proporcionam estimativas mais confiáveis de divergência genética do que locos com menor polimorfismo. Kalinowski (2002) comenta ainda que o número observado de alelos constitui um indicador adequado do coeficiente de variação de estimativas de divergência genética. Isso indica que o fator limitante da consistência dessas estimativas é o número total de alelos e não necessariamente o número de locos. Embora o número de locos utilizados no presente estudo tenha sido reduzido, o alto conteúdo de informação genética observado mostrou-se adequado para a avaliação da estrutura populacional.

De modo geral, como já observado pelas estimativas de $\hat{R}_{S T}$, o fluxo gênico estimado pelo método indireto $(\mathrm{Nm})$ mostra que o número de migrantes por geração trocados

Tabela 5. Estimativas de divergência genética $\left(\hat{R}_{S T}-\right.$ diagonais inferiores) e fluxo gênico aparente ( $\mathrm{Nm}$-diagonais superiores) entre pares de populações de adultos e regenerantes de $C$. langsdorffii, no Pontal do Paranapanema, SP. (MD = Parque Estadual do Morro do Diabo; $\mathrm{MC}=$ Madre Cristina; $\mathrm{TU}=$ Tucano).

Table 5. Estimates of genetic divergence ( $\hat{R}_{S T}-$ low diagonals) and apparent gene flow ( $\mathrm{Nm}$ - upper diagonals) between pairs of adult and sapling Copaifera langsdorffii populations in "Pontal do Paranapanema", SP. (MD = "Parque Estadual do Morro do Diabo"; MC = "Madre Cristina"; TU = "Tucano").

\begin{tabular}{llll}
\hline & \multicolumn{1}{c}{ MD } & TU & MC \\
\hline Adultos & & & \\
MD & - & 15,65 & 4,50 \\
TU & $0,0157^{\mathrm{ns}}$ & - & 6,53 \\
MC & $0,0527^{* *}$ & $0,0369^{*}$ & - \\
Regenerantes & & & \\
MD & - & 2,50 & 5,90 \\
TU & $0,0910^{* *}$ & - & 2,24 \\
MC & $0,0407^{* *}$ & $0,1005^{* *}$ & - \\
\hline
\end{tabular}

ns = não significativo (not significant) $; *$ = significativo a $5 \%$ (significant at $5 \%) ; *=$ significativo a $1 \%($ significant at $1 \%)$. 
entre as populações é elevado, mas foi consideravelmente restringido após a fragmentação, especialmente entre a população mais isolada (Tucano) (tabela 5). Considerando-se o conjunto de populações, a estimativa de fluxo gênico $(\mathrm{Nm})$ entre os adultos foi de 6,93 migrantes por geração e entre os regenerantes foi de 3,06.

A pequena diferenciação genética entre as populações adultas avaliadas nesse estudo é consistente com as altas taxas de cruzamento que as espécies arbóreas tropicais costumam apresentar (Hall et al. 1996) e são coerentes com a biologia reprodutiva da espécie. Um estudo sobre o sistema de reprodução de Copaifera langsdorffii (Oliveira et al. 2002) mostrou que a espécie reproduz-se predominantemente por fecundação cruzada $\left(\hat{t}_{m}=0,917\right.$ $\pm 0,041)$ e que os cruzamentos entre indivíduos aparentados não são comuns $\left(\hat{t}_{m}-\hat{t}_{s}=0,040 \pm 0,023\right)$. $O$ transporte de pólen é efetivado por abelhas e a dispersão de sementes é zoocórica. Apenas as aves de grande tamanho dispersam as sementes a longas distâncias. As aves de pequeno porte regurgitam as sementes próximas à planta-mãe (Motta Junior \& Lombardi 1990), restringindo o fluxo gênico entre as populações e entre árvores distantes. Adicionalmente, há frutos que não são consumidos pela fauna e apenas caem ao solo, ficando sob a copa da planta-mãe. As formigas desempenham um importante papel na dispersão secundária das sementes de $C$. langsdorffii. Elas carregam as sementes por centenas de metros dentro de cada fragmento, fazendo com que a endogamia entre os regenerantes não seja muito diferente da observada nos adultos.

A fragmentação florestal está causando restrição no fluxo gênico entre populações, especialmente em relação ao fragmento mais distante (Tucano). Antes da supressão da vegetação florestal, a distância de $8 \mathrm{~km}$ entre os locais de coleta das populações do parque e de Tucano possibilitava o fluxo gênico, fazendo com que os adultos amostrados nessas duas áreas fizessem parte de uma única população panmítica. O maior isolamento espacial do fragmento Tucano pode estar resultando na maior diferenciação genética dos regenerantes dessa população. Os espaços não florestados entre as populações de C. langsdorffii podem dificultar o movimento das abelhas polinizadoras. As aves de grande porte não têm mais sido frequentemente observadas na região do Pontal do Paranapanema. Dessa forma, o aumento na divergência genética entre as populações regenerantes pode ser decorrente da limitação da dispersão de pólen e sementes em longas distâncias.

Espécies arbóreas longevas que apresentam banco de sementes ou plântulas que sobrevivem por muito tempo ficam protegidas dos efeitos da fragmentação por décadas ou séculos (Templeton \& Levin 1979, Cabin
1996). Alterações recentes no fluxo gênico e nas taxas de cruzamento das populações fragmentadas correm o risco de não serem detectados por um longo período (England et al. 2002). A fragmentação da região é recente, tendo sido iniciada na década de 1920, sendo o tempo muito curto para que a deriva genética e a seleção provoquem diferença tão acentuada entre as populações de regenerantes. Deve ser considerada também a distância geográfica entre as populações, uma vez que o fragmento Tucano dista oito quilômetros do parque e o fragmento Madre Cristina está separado deste apenas pela largura de uma estrada, permitindo, mesmo que em menor intensidade, o fluxo gênico entre esses fragmentos e a floresta contínua do Parque Estadual do Morro do Diabo.

O florescimento supra-anual da espécie (Pedroni et al. 2002), a falta de sincronia completa e a ocorrência de períodos com baixa freqüência de florescimento podem intensificar os efeitos da fragmentação em decorrência do menor tamanho efetivo populacional nos fragmentos pequenos (Hall et al. 1996). Esses efeitos já foram observados em outra espécie arbórea tropical, Pithecellobium elegans (Hall et al. 1996). Cabe ressaltar que, embora a endogamia tenha sido elevada, a diversidade genética nessas populações foi expressiva, uma vez que a amostragem envolve indivíduos que foram gerados em diversos períodos de florescimento.

Faz-se necessária a tomada de providências no sentido de aumentar o fluxo gênico, promovendo assim uma mistura entre os alelos das populações remanescentes do Pontal do Paranapanema. A conectividade facilitaria a manutenção da diversidade genética. A estratégia para aumentar a conectividade consiste em plantar estrategicamente indivíduos de C. langsdorffii entre estas populações, no intuito de se comportarem como trampolins ecológicos, gerando conectividade entre remanescentes florestais. Uma alternativa para a região do Pontal do Paranapanema, a qual é ocupada por extensas fazendas e assentamentos de Reforma Agrária, seria o plantio de C. langsdorffii em módulos de sistemas agroflorestais pelos agricultores assentados na região. Módulos de sistemas agroflorestais têm sido implantados em roças de alguns proprietários rurais assentados, tanto próximos ao fragmento Tucano quanto ao fragmento Madre Cristina, sendo uma iniciativa que deve ser incentivada.

Agradecimentos - Os autores agradecem Elza M. Ferraz, Gelson D. Fernandes, Glauce Paschoalini e Rafael Bortoletto pelo apoio técnico no laboratório e Aderson R. B. Gomes (COCAMP), Edilson Soares (Assentamento Tucano) e José Gomes (PEMD) pelo auxílio no trabalho de campo. Alexandre 
M. Sebbenn (Instituto Florestal/SP) e Lúcia Helena de O. Wadt (Embrapa Acre) contribuíram com sugestões valorosas em versões prévias desse manuscrito. A pesquisa foi financiada por International Plant Genetic Resources Institute (IPGRI) Roma. Bolsas de estudos de Doutoramento (CNPq) para Karina Martins e de Pós-Doutoramento (FAPESP) para Fernanda Amato Gaiotto.

\section{Referências bibliográficas}

ALDRICH, P.R., HAMRICK, J.L., CHAVARRIAGE, P. \& KOCHERT, G. 1998. Microsatellite analysis of demographic genetic structure in fragmented populations of the tropical tree Symphonia globulifera. Molecular Ecology 7:933-944.

BALLOUX, F. \& LUGON-MOULIN, N. 2002. The estimation of population differentiation with microssatelite markers. Molecular Ecology 11:155-165.

BROOKFIELD, J.F.Y. 1996. A simple new method for estimating null allele frequency from heterozygote deficiency. Molecular Ecology 5:453-455

CABIN, R.J. 1996. Genetic comparisons of seed bank and seedling populations of a perennial desert mustard, Lesquerella fendleri. Evolution 50:1830-1841.

CARLINI-GARCIA, L.A., VENCOVSKY, R. \& COELHO, A.S.G. 2001. Método bootstrap aplicados em níveis de reamostragem na estimação de parâmetros genéticos populacionais. Scientia Agricola 58:785-793.

CERVANTES-MARTINEZ, C., BROWN, J.S., SCHNELL, R., MOTAMAYOR, J.C., MEEROW, A.W. \& ZHANG, D. 2006. A computer simulation study on the number of loci and trees required to estimate genetic variability in cacao (Theobroma cacao L.). Tree Genetics and Genomics 2:152-164.

CHASE, M., KESSELI, R. \& BAWA, K. 1996. Microsatellite markers for population and conservation genetics of tropical trees. American Journal of Botany 83:51-57.

CIAMPI, A.Y. \& GRATTAPAGLIA, D. 2001. Variabilidade genética em populações de copaíba (Copaifera langsdorffii Desf. - Caesaelpiniaceae) estimada com polimorfismos de AFLP, microssatélites e seqüenciamento de cpDNA. Embrapa Recursos Genéticos e Biotecnologia, Boletim de Pesquisa e Desenvolvimento, 12:1-32.

CIAMPI, A.Y., BRONDANI, R.P.V. \& GRATTAPAGLIA, D. 2000. Desenvolvimento de marcadores microssatélites para Copaifera langsdorffii Desf. (copaíba) Leguminosae - Caesalpinoideae e otimização de sistemas fluorescentes de genotipagem multiloco. Embrapa Recursos Genéticos e Biotecnologia, Boletim de Pesquisa, 16:1-40.

COELHO, A.S.G. 2000. BOOD v. 2.0. Avaliação de dendrogramas baseados em estimativas de distâncias/ similaridades genéticas através do procedimento de bootstrap. Laboratório de Genética Vegetal, DBG/ICB/ UFG.
CRESTANA, C.S.M. \& KAGEYAMA, P.Y. 1989. Biologia da polinização de Copaifera langsdorffii Desf. (Leguminosae-Caesalpinoideae), o "óleo-de-copaíba". Revista do Instituto Florestal 1:201-214.

CRESTE, C., TULMANN-NETO, A. \& FIGUEIRA, A. 2001. Detection of single sequence repeat polymorphisms in denaturing polyacrylamide sequencing gels by silver staining. Plant Molecular Biology Reporter 19:299-306.

DAYANANDAN, S., DOLE, J., BAWA, K. \& KESSELI, R. 1999. Population structure delineated with microsatellite markers in fragmented populations of a tropical tree, Carapa guianensis (Meliaceae). Molecular Ecology 8:1585-1592.

DEAN, W. 1996. A ferro e fogo: a história e a devastação da Mata Atlântica Brasileira. Companhia das Letras, São Paulo.

DOYLE, J.J. \& DOYLE, J.S. 1987. Isolation of plant DNA from fresh tissue. Focus 12:13-15.

ENGLAND, P.R., USHER, A.V., WHELAN, R.J. \& AYRE, D.J. 2002. Microsatellite diversity and genetic structure of fragmented populations of the rare, fire-dependent shrub Grevillea macleayana. Molecular Ecology 11:967-977.

FREITAS, C.V. \& OLIVEIRA, P.E. 2002. Biologia reprodutiva de Copaifera langsdorffii Desf. (Leguminosae, Caesalpinioideae). Revista Brasileira de Botânica 25: 311-321.

GOODMAN, S.J. 1997. $\mathrm{R}_{\mathrm{ST}}$ Calc: a collection of computer programs for calculating estimates of genetic differentiation from microsatellite data and determining their significance. Molecular Ecology 6:881-885.

HALL, P., WALKER, S. \& BAWA, K. 1996. Effect of forest fragmentation on genetic diversity and mating system in a tropical tree, Pithecellobium elegans. Conservation Biology 10:757-768.

JARNE, P. \& LAGODA, P.J.L. 1996. Microsatellites, from molecules to populations and back. Trends in Ecology and Evolution 11:424-429.

KALINOWSKI, S.T. 2002. How many alleles per locus should be used to estimate genetic distances? Heredity 88:62-65.

LEBERG, P.L. 2002. Estimating allelic richness: effects of sample size and bottlenecks. Molecular Ecology 11: 2445-2449.

LEWIS, P.O. \& ZAYKIN, D. 2000. Genetic Data Analysis: computer program for the analysis of allelic data. Version 1.0. http://alleyn.eeb.uconn.edu/gda/2000 (acesso em 09 de junho de 2002).

MILLER, M.P. 1997. TFPGA - Tools for Population Genetic Analyses: A Windows ${ }^{\circledR}$ program for the analyses of allozymes and molecular population genetic data. Departament of Biological Sciences, Northern Arizona University.

MARSHALL, T.C., SLATE, J., KRUUK, L. \& PEMBERTON, J.M. 1998. Statistical confidence for likelihood-based paternity inference in natural populations. Molecular Ecology 7:639-655. 
MOTTA JUNIOR, J.C. \& LOMBARDI, J.A. 1990. Aves como agentes dispersores de copaíba (Copaifera langsdorffii, Caesalpiniaceae) em São Carlos, estado de São Paulo. Ararajuba 1:105-106.

NEI, M. 1978. Estimation of average heterozigosity and genetic distance from a small number of individuals. Genetics 89:583-590.

OLIVEIRA, A.F., CARVALHO, D. \& ROSADO, S.C.S. 2002. Taxa de cruzamento e sistema reprodutivo de uma população natural de Copaifera langsdorffii Desf. na região de Lavras (MG) por meio de isoenzimas. Revista Brasileira de Botânica 25:331-338.

OOSTERHOUT, C.V., HUTCHINSON, W.F., WILLS, D.P.M. \& SHIPLEY, P. 2004. Micro-checker: software for identifying and correcting genotyping errors in microsatellite data. Molecular Ecology Notes 4:535-538.

PEDRONI, F., SANCHEZ, M. \& SANTOS, F.A.M. 2002. Fenologia da copaíba (Copaifera langsdorffii Desf. Leguminosae, Caesalpinoideae) em uma floresta semidecídua no sudeste do Brasil. Revista Brasileira de Botânica 25:183-194.

SAMPAIO, P.T.B. 2000. Copaíba. In: Biodiversidade amazônica: exemplos e estratégias de utilização (W. Clay, P.T. Sampaio \& C.R. Clement, eds.). INPA, Manaus, p.207-215.
SHAPCOTT, A. 1998. The patterns of genetic diversity in Carpentaria acuminata (Arecaceae), and rainforest history in northern Australia. Molecular Ecology 7:833-847.

TEMPLETON, A.R. \& LEVIN, D.A. 1979. Evolutionary consequences of seedbanks. American Naturalist 114: 232-249.

VEIGA JÚNIOR, V.F. \& PINTO, A.C. 2002. O gênero Copaifera L. Química Nova 25:273-286.

WHITE, G.M., BOSHIER, D.H. \& POWELL, W. 1999. Genetic variation within a fragmented population of Swietenia humilis Zucc. Molecular Ecology 8:1899-1909.

WILLIAMS, B.L., BRAWN, J.D. \& PAIGE, K.N. 2003. Landscape scale genetic effects of habitat fragmentation on a high gene flow species: Speyeria idalia (Nymphalidae). Molecular Ecology 12:11-20.

WRIGHT, S. 1951. The genetical structure of populations. Annals of Eugenics 15:323-354.

YOUNG, A.G., MERRIAM, H.G. \& WARWICK, S.I. 1993. The effects of forest fragmentation on genetic variation in Acer saccharum Marsh. (sugar maple) populations. Heredity 71:277-289.

YOUNG, A.G., BOYLE, T. \& BROWN, T. 1996. The population genetic consequences of habitat fragmentation for plants. Trends in Ecology \& Evolution 11:413-418. 